$$
u(0, y)=y / 2-\log \left(1+e^{y}\right) .
$$

The solution of this problem is $u(x, y)=(x+y) / 2-\log \left(e^{x}+e^{y}\right)$.

Taking $h$ as 0.05 , we have written a program in Fortran for the CDC 1604 computer at the University of California, San Diego and have found the following crrors for the computation of $u$ at the points $(x, y)$ given below. By error we mean here the relative error, i.e.,

$$
\text { error }=\mid \text { (true value }- \text { approximate value }) / \text { true value } \mid \text {. }
$$

As a concluding remark, we wish to point out that the techniques used here can be applied to the more general equation $u_{x y}=f\left(x, y, u, u_{x}, u_{y}\right)$. In this case, one could use the quadrature formula used above and the Moore-RungeKutta method to estimate the values of $u, u_{x}, u_{y}$ at the quadrature evaluation points.

I am especially indebted to Prof. P. C. Hammer and A. H. Stroud for many discussions on the general techniques of numerical integration in more than one variable.

University of California, San Diego

La Jolla, California

1. P. C. Hammer \& J. W. Hollingsworth, "Trapezoidal methods of approximating solutions of differential equations," $M T A C$, v. 9, 1955, p. 92-96.

2. D. Morrison \& L. STOLLER, "A method for the numerical integration of ordinary differential equations," $M T A C$, v. 12,1958 , p. 269-272.

3. P. C. Hammer \& A. W. Wymore, "Numerical evaluation of multiple integrals I," MTAC, v. 11,1957, p. 59-67.

4. J. AlbRechT \& L. Collatz, "Zur numerischen Auswertung mehrdimensionaler Integrale," Z. Angew. Math. Mech., v. 38, 1958, p. 8.

5. R. H. Moore, "A Runge-Kutta procedure for the Goursat problem in hyperbolic partial differential equations," Arch. Rational Mech. Anal., v. 7, 1961, p. 37-63.

6. W. Törnig, "Zur numerischen Behandlung von Anfangswertproblemen partieller hyperbolischer Differentialgleichungen zweiter Ordnung in zwei unabhangigen Veranderlichen, I," Arch. Rational Mech. Anal., v. 4, 1960, p. 428-445.

7. C. Jordan, Cours d'Analyse, Gauthier-Villars, Paris, Third Edition, v. 3, 1915, p. 369371.

8. W. WALTER, "Fehlerabschätzungen bei hyperbolischen Differentialgleichungen," Arch. Rational Mech. Anal., v. 7, 1961.p. 249-272.

9. P. Henrici, Discrete Variable Methods in Ordinary Differential Equations, John Wiley and Sons, New York, 1962.

\title{
Gauss Elimination for Singular Matrices
}

\section{By George Shapiro}

1. Introduction. Several variations of a method of successive elimination, associated with the name of Gauss, are frequently used to solve systems of linear equations or to invert a matrix. Multiplication of the inverse matrix by the determinant of the original matrix (which is readily available as an intermediate result of the Gauss elimination) yields the adjoint of the original matrix.

Recently, the use of modular or residue class arithmetic systems for highspeed computers has been considered [1]. In such systems, an integer is represented

Received November 13, 1962, revised March 18, 1963. The research leading to this paper was sponsored by the Electronic Technology Laboratory of the USAF Aeronautical systems Division. 
by the set of its residues modulo various primes; that is, by a set of remainders obtained when the integer is divided by each of the primes. (More generally, pairwise relatively prime moduli have been considered but prime moduli are required for the problems to be discussed here.) The details of the arithmetic of these systems are of no interest here; it should be noted, however, that only integers may be treated directly.

The inverse of a matrix $A$ with integer entries may have fractional entries; the solution of a set of linear equations $A x=y$ ( $x$ and $y$ column vectors) with integer coefficients may be fractional; therefore, in modular arithmetic systems it is necessary to recast these problems so as to have integer solutions. In each case, the product of $|A|$, the determinant of $A$, and the solution is a set of integers. (In many cases, a factor less than $|A|$ may be used for this purpose, but considerable computation is involved in finding this factor.) Thus inversion of a matrix is replaced by finding its adjoint, $A^{*}$, and the variable $x$ of the set of equations is replaced by $z$ which satisfies

$$
z=|A| x=A^{*} y \text {. }
$$

When $|A|$ is divisible by one or more of the prime moduli of the system, $A$ while in general non-singular, is singular as far as computations modulo those primes are concerned. Thus, the problem of finding $A^{*}$ for singular as well as non-singular $A$, is fundamental to linear algebra in modular arithmetic systems. The author is not aware of a significant use for the adjoint of a singular matrix in conventional arithmetic systems.

While the adjoint of a singular matrix is well-defined, the Gauss process breaks down when applied to a singular matrix. Thus, it has been necessary to use an alternate procedure to find the adjoint of a singular matrix. One such procedure is described in [2]. However, it necessitates a special treatment of singular matrices. In the sequel, a simple extension of the Gauss method which permits the calculation of the adjoint of a singular matrix is described. Applied to a system of linear equations with singular coefficient matrix in the same fashion as the usual Gauss technique it leads directly to the solution in the form (1).

2. Gauss Elimination. To avoid confusion, one standard variant of the Gauss elimination process will be described and will hereafter be considered as the Gauss process. The extension works equally well with other variants; obvious minor modifications to the proof of its validity are required.

To invert the $n \times n$ matrix $A$ the process is as follows. The $n \times n$ identity, $I$, is adjoined to $A$ to form an $n \times 2 n$ matrix $(A, I)$. The non-singular operations of row interchanges and elementary row operations (multiplication of a row by a nonzero constant and addition of a multiple of a row to another row) are performed to bring $A$ first to upper triangular form (the forward course) and then to complete the transformation of $A$ to the identity, $I$, (the return course). At this point, $I$ in the augmented matrix has been transformed to $A^{-1}$ and the determinant of $A$ is the reciprocal of the product of the factors used as row multipliers times $(-1)^{r}$, where $r$ is the number of row interchanges performed. The adjoint of $A$ is given by $A^{*}=|A| A^{-1}$.

The forward course may always be completed; when $A$ is singular, one or more 
rows of the transform of $A$ will be 0 . The return course, which starts with the $n$th row of $A$, and uses only elementary row operations (no row interchanges) can proceed only to the point where addition of an (infinite) multiple of a zero row to other rows is required.

For definiteness, it is assumed that by this point, all diagonal elements of the transform of $A$ are either 0 or 1 . If not, the rows of the augmented matrix are each multiplied by the reciprocal of the corresponding non-zero diagonal element. The product, $b$, of the reciprocals of all the row multipliers and $(-1)^{r}$ is required. At this point then, the matrix $(A, I)$ has been transformed to $(T, S)$ where

$$
\operatorname{det} S=1 / b \neq 0
$$

and $T$ has at least one row of zeros. Since the same operations which transformed $A$ to $T$ transformed $I$ to $S$,

$$
S A=T
$$

The same process is followed to solve a set of linear equations except that the column $y$ is adjoined to $A$ to form the $n \times(n+1)$ matrix $(A, y)$. This process breaks down at the same point.

3. Extended Gauss Elimination. The extension is simply the addition of the following operation, $E$, to those previously used. When a row of zeros, say the $i$ th, $i$ is encountered in the transform of $A$, the diagonal element of that row is changed to 1 , and in the augmented portion of the matrix all other rows are changed to 0 , the ith row being unchanged.

This operation, $E$, treats the portions $T$ and $S$ or $T$ and $S y$ of the transformed augmented matrix dissimilarly. Let $D_{i}$ be the $n \times n$ matrix with a 1 in the $(i, i)$ position and 0 elsewhere. Then $E$ is simply:

$$
\begin{aligned}
& T \rightarrow T+D_{i} \\
& S \rightarrow D_{i} S
\end{aligned}
$$

or

$$
(S y) \rightarrow D_{i}(S y)
$$

Thereafter the ordinary Gauss process proceeds. Since all diagonal elements used in the Gauss process are 1, only the operation of addition of a multiple of one row to another is required. This operation has determinant 1.

If another 0 diagonal element, say the $j$ th, is encountered, the operation $E$ is repeated. After a finite number of such operations (at most $n$ ) the augmented matrix of the inversion problem is in the form $(I, W)$. Then,

$$
b W=A^{*} \text {. }
$$

For the problem of the solution of the set of linear equations, $W$ is not displayed explicitly; the augmented portion of the matrix is $(W y)$; then

$$
b(W y)=A^{*} y=z
$$

from (1). 
It remains to show that inclusion of the operation $E$ in the above procedure leads to $A^{*}$.

From (3) it follows that

$$
A^{*} S^{*}=T^{*}
$$

Multiplying on the right by $\mathrm{S}$ and using (2) yields

$$
A^{*}=b T^{*} S \text {. }
$$

Since the $i$ th row of $T$ is 0 , the cofactor of each element of $T$ not in the $i$ th row is 0 so that $T^{*}$ differs from 0 only in the $i$ th column. But then $T^{*} S$ depends only on the $i$ th row of $S$ so that $S$ may be replaced by $D_{i} S$ which leaves the $i$ th row of $S$ unchanged; thus,

$$
A^{*}=b T^{*}\left(D_{i} S\right) \text {. }
$$

(This relation verifies the known result that the rows of $A^{*}$ are proportional to the $i$ th row of $S$.)

Since $D_{i} S$ differs from 0 only in the $i$ th row, the product $T^{*}\left(D_{i} S\right)$ depends only on the $i$ th column of $T^{*}$; this column is independent of the $i$ th row of $T$ so that $T$ may be replaced by $T+D_{i}$. Hence

$$
A^{*}=b\left(T+D_{i}\right)^{*}\left(D_{i} S\right) \text {. }
$$

If $T+D_{i}$ is non-singular, Gauss elimination on the augmented matrix

$$
\left(T+D_{i}, D_{i} S\right)
$$

can be completed leading to a matrix $(I, W)$. Clearly the transformation of $T+D_{i}$ to $I$ is accomplished by $\left(T+D_{i}\right)^{-1}$; since $T+D_{i}$ is a triangular matrix with diagonal elements 1 , its determinant is 1 so that

$$
\left(T+D_{i}\right)^{-1}=\left(T+D_{i}\right)^{*}
$$

and

$$
W=\left(T+D_{i}\right)^{*}\left(D_{i} S\right)
$$

Substituting in (4):

$$
A^{*}=b W
$$

as required.

A proof for the case $T+D_{i}$ singular may be had for example, by induction on the number of 0 diagonal elements of $T$. It may be noted that if 2 or more rows of the transform of $A$ are simultaneously 0 , say the $i$ th and $j$ th, then $S$ may be replaced by

$$
D_{i} D_{j} S=0 .
$$

Since any linear transformation of 0 yields 0 , the known result,

$$
A^{*}=0
$$

when $A$ is multiply degenerate, is obtained. Unfortunately, no simple a priori test for multiple degeneracy is available; the following example shows that the 
operation $E$ may be required twice for a singly degenerate matrix:

$$
A=\left(\begin{array}{lll}
1 & 0 & 0 \\
0 & 0 & 1 \\
0 & 0 & 0
\end{array}\right)
$$

Westinghouse Electric Corporation

Baltimore, Maryland

1. H. Garner, "The residue number system," IRE Trans. on Electronic Computers, Vol. EC-8, pp 140-147, June 1959.

2. Frazer, Duncan \& Collar, Elementary Matrices, Cambridge University Press, 1960.

\section{A Remark on a Paper of Bateman and Horn}

\section{By A. Schinzel}

Let $f_{1}, f_{2}, \cdots, f_{k}$ be distinct irreducible polynomials with integral coefficients and the highest coefficient positive, such that $f(x)=f_{1}(x) f_{2}(x) \cdots f_{k}(x)$ has no fixed divisor $>1$. Denote by $P(N)$ the number of positive integers $x \leqq N$ such that all numbers $f_{1}(x), f_{2}(x), \cdots, f_{k}(x)$ are primes.

P. T. Bateman and R. A. Horn [1] recently gave the heuristic asymptotic formula for $P(N)$ :

$$
P(N) \sim \frac{N}{\log ^{k} N}\left(h_{1} h_{2} \cdots h_{k}\right)^{-1} \prod_{p}\left(1-\frac{\omega(p)}{p}\right)\left(1-\frac{1}{p}\right)^{-k},
$$

where $h_{i}$ is the degree of $f_{i}$ and $\omega(p)$ is the number of solutions of the congruence $f(x) \equiv 0(\bmod p)$.

Formula (1) contains as particular cases six conjectures from a well-known paper of Hardy and Littlewood [3] called by the latter Conjectures B, D, E, F, $\mathrm{K}, \mathrm{P}$, as well as their conditional theorem $\mathrm{X} 1$. This is evident except for Conjecture $\mathrm{D}$ which concerns the number of solutions of the equation

$$
a p-b p^{\prime}=k \quad(a>0, b>0,(a, b)=1)
$$

in primes $p, p^{\prime}$ with $p \leqq n$. In order to apply formula (1) here one should put $f_{1}(x)=u_{0}+b x, f_{2}(x)=v_{0}+a x, N=\frac{n-u_{0}}{b}$, where $u_{0}, v_{0}$ are fixed integers such that $a u_{0}-b v_{0}=k$.

Conjectures denoted by Hardy and Littlewood by J, M, N are of distinctly different character; besides the first has been proved by S. Chowla [2] and Ju. V. Linnik [4]. Conjecture A, (a strong form of Goldbach's Conjecture), is a particular case of $\mathrm{C}$, Conjectures $\mathrm{H}$ and $\mathrm{I}$ are particular cases of $\mathrm{G}$. It remains therefore to consider Conjectures $\mathrm{C}, \mathrm{G}, \mathrm{L}$, which are, according to Hardy and Littlewood, conjugate to Conjectures $\mathrm{D}, \mathrm{F}, \mathrm{K}$ respectively. We quote them below for the convenience of a reader, with slight changes in the notation (e.g. $p, p^{\prime}$ denote primes).

Received February 4, 1963, revised May 13, 1963. 